\title{
Efeito do residual no solo de nicosulfuron isolado e em mistura com atrazine sobre culturas agrícolas subsequentes
}

\author{
Carryover effect of nicosulfuron alone and tank mixture with atrazine on \\ subsequent crops
}

\author{
Fernando Tadeu de Carvalho ${ }^{1}$, Talita Breda Moretti ${ }^{2}$, Priscila Alves de Souza ${ }^{3}$
}

\begin{abstract}
Resumo - O trabalho foi desenvolvido com o objetivo de avaliar o efeito do residual no solo dos herbicidas nicosulfuron isolado e nicosulfuron + atrazine sobre as culturas de soja, feijão, algodão, pepino, girassol e arroz, semeadas em diferentes períodos após a aplicação dos herbicidas. O experimento foi desenvolvido no período de novembro/2008 a abril/2009, na FEPUNESP, no município de Selvíria/MS, em solo com textura argilosa. O experimento foi conduzido sob o sistema de plantio convencional com as culturas sendo semeadas em espaçamento de $0,5 \mathrm{~m}$. O delineamento foi em blocos ao acaso, com 13 tratamentos e 4 repetições, por cultura. As parcelas foram dimensionadas com $15 \mathrm{~m}^{2}(3 \mathrm{~m} \times 5 \mathrm{~m})$, com $10 \mathrm{~m}^{2}$ centrais de área aplicada. Os tratamentos foram constituídos pelos herbicidas nicosulfuron + atrazine $\left(30 \mathrm{~g}+1500 \mathrm{~g} \mathrm{ha}^{-1}\right)$ e nicosulfuron $\left(60 \mathrm{~g} \mathrm{ha}^{-1}\right)$ aplicados ao solo aos 75, 60, 45, 30, $15 \mathrm{e}$ 7 dias antes da semeadura das culturas e uma testemunha sem herbicida. Considerando-se os resultados obtidos concluiu-se que as culturas do feijão (Carioca), pepino (Caipira-Esmeralda) e girassol (Dow M734-G3) podem ser semeadas após 30 dias da aplicação de nicosulfuron + atrazine e nicosulfuron isolado. A cultivar de soja Carrera pode ser semeada 15 e 30 dias após a aplicação de nicosulfuron + atrazine e nicosulfuron isolado, respectivamente. O algodão (DeltaOpal) pode ser semeado após 60 e 45 dias da aplicação de nicosulfuron + atrazine e nicosulfuron isolado, respectivamente. Já o arroz (Primavera) pode ser semeado após 60 dias da aplicação de nicosulfuron + atrazine e nicosulfuron isolado.
\end{abstract}

Palavras-chave: Accent, Atrazinax 500, carryover, herbicidas.

\begin{abstract}
The work was developed with the objective of evaluating the carry over effect in soil of nicosulfuron herbicide isolated and nicosulfuron + atrazine over soybean, bean, cotton, cucumber, sunflower and rice crops, sown in different periods after herbicides application. The experiment was carried out from November/2008 to April/2009, at FEP-UNESP, in Selviria, County/MS, with clayey soil. The experiment was conducted under conventional tillage with crops sown in $0.5 \mathrm{~cm}$ spacing. The experimental design was randomized complete block with 13 treatments and 4 replications per crop. Plots were scaled with $15 \mathrm{~m}^{2}(3 \mathrm{~m} \times 5 \mathrm{~m})$, with $10 \mathrm{~m}^{2}$ of applied central area. Treatments consisted of nicosulfuron + atrazine $\left(30 \mathrm{~g}+1500 \mathrm{~g} \cdot \mathrm{ha}^{-1}\right)$ and nicosulfuron $\left(60 \mathrm{~g} \cdot \mathrm{ha}^{-1}\right)$ herbicides applied to the soil at $75,60,45,30,15$ and 7 days before crop sowing and a check without herbicide. Considering the obtained results, it was concluded that
\end{abstract}

\footnotetext{
${ }^{1}$ Professor Dr., DBZ-FEIS-UNESP, Ilha Solteira/SP, Brasil. E-mail: ftadeu@ bio.feis.unesp.br;

${ }^{2}$ Pós-graduanda de Agronomia, FEIS-UNESP, Ilha Solteira/SP;

${ }^{3}$ Aluna do curso de Agronomia Universidade Castelo Branco - UNICASTELO, Fernandópolis/SP.
}

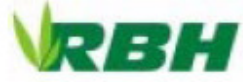


bean (Carioca cultivar), cucumber (Caipira-Esmeralda cultivar) and sunflower (Doe M734-G3 genotype) crops may be sown 30 days after atrazine + nicosulfuron application, as soon as isolated nicosulfuron. Soybean crop (Carrera cultivar) may be sown between 15 and 30 days after nicosulfuron + atrazine application and isolated nicosulfuron, respectively. Cotton crop (DeltaOpal cultivar), may be sown after 60 and 45 days of nicosulfuron + atrazine and isolated nicosulfuron application, respectively. However, rice crop (Primavera cultivar) may be sown 60 days after nicosulfuron + atrazine and isolated nicosulfuron application.

Key-words: Accent, Atrazinax 500, carryover, herbicides.

\section{Introdução}

A cultura do milho está entre as mais importantes do Brasil com uma área plantada em torno de 14 milhões de hectares $\mathrm{e}$ produtividade média de $3.670 \mathrm{~kg} \mathrm{ha}^{-1}$ (IBGE, 2009). As condições edafoclimáticas destacam o Brasil como um país de grande potencial para a cultura do milho, entretanto, o clima tropical é também muito propício à ocorrência de uma grande quantidade de plantas daninhas, que interferem no desenvolvimento $e$ na produtividade da cultura.

Os efeitos negativos detectados no crescimento e produtividade da cultura do milho, devido à presença das plantas daninhas, já foram observados por vários autores (Carvalho \& Galli, 1993; Velini et al., 1993; Silva et al., 1993; Fancelli \& Dourado Neto, 2000).

Existem diferentes métodos para $\mathrm{o}$ controle das plantas daninhas. Nas regiões tecnificadas de cultivo do milho o controle químico é o que tem sido mais utilizado através, principalmente, dos herbicidas atrazine na pré-emergência e do nicosulfuron na pósemergência. $\mathrm{O}$ atrazine pertence ao grupo químico das triazinas, é um herbicida residual totalmente seletivo para a cultura do milho, indicado para o controle de plantas daninhas de folhas largas e algumas gramíneas em pré ou pós-emergência inicial (López-Ovejero et al., 2003). O nicosulfuron pertence ao grupo químico das sulfoniluréias, é um herbicida pósemergente recomendado para a cultura do milho no controle de gramíneas e algumas folhas largas (Rodrigues \& Almeida, 2005; Andrei, 2005; Lorenzi et al., 2006).

O manejo de plantas daninhas através da aplicação de herbicidas é uma característica do cultivo agrícola tecnificado, entretanto é sempre importante que seja observado e respeitado o período residual dos herbicidas para que as culturas subseqüentes não sejam afetadas. O parâmetro utilizado para estimar a persistência do produto no solo é a meia-vida que, segundo Rodrigues \& Almeida (2005), é de 55 dias para atrazine e de 21 dias para nicosulfuron, porém como as culturas respondem diferentemente quanto à sensibilidade aos herbicidas, são importantes os trabalhos de pesquisa que analisem as diferentes situações.

López-Ovejero et al. (2003) citam que além eficiência no controle de plantas daninhas, é importante saber a influência que os herbicidas usados tanto em pré como em pós-emergência causam no desenvolvimento da cultura de milho e das culturas subsequentes.

Ressalta-se que a cultura do milho é semeada em diferentes épocas do ano, nas modalidades de milho normal e safrinha, e que é possível que o efeito residual dos herbicidas, venha a afetar o crescimento de culturas subsequentes. Desta forma, o presente trabalho foi desenvolvido com o objetivo de avaliar o efeito do residual no solo dos herbicidas nicosulfuron isolado e nicosulfuron + atrazine sobre as culturas de soja, feijão, algodão, 
pepino, girassol e arroz, semeadas em diferentes períodos após a aplicação dos herbicidas.

\section{Material e Métodos}

$\mathrm{O}$ experimento foi realizado no período de novembro/2008 a abril/2009, em área irrigada da Fazenda de Ensino e Pesquisa (FEP) da UNESP - FEIS, nas coordenadas S $20^{\circ} 20^{\prime} 34,3$ " e WO 51 $24^{\prime} 01,6$ ", com 335 metros de altitude, localizada no município de Selvíria, MS, enquadrada em região de cerrado, na região nordeste do Mato Grosso do Sul. O solo da área experimental é classificado como Latossolo Vermelho-Escuro textura argilosa, com $40 \%$ de argila, $52 \%$ de areia e $8 \%$ de silte.

As culturas testadas no experimento foram a soja (Glycine max) cultivar Carrera (precoce); feijão (Phaseolus vulgaris) variedade carioca; algodão (Gossypium hirsutum) variedade Delta-Opal; pepino (Cucumis sativus) cultivar Caipira-Esmeralda; girassol (Helianthus annus) híbrido Dow M734-G3 e arroz (Oryza sativa) variedade Primavera. As culturas de soja, feijão, algodão, girassol e arroz, foram semeadas mecanicamente no sistema de plantio convencional, em solo nú e destorroado, em $25 / 01 / 2008$, no espaçamento de $0,50 \mathrm{~m}$ entrelinhas.

A semeadura de pepino foi realizada manualmente, nas mesmas condições de solo e espaçamento. $\mathrm{O}$ delineamento experimental adotado foi o de blocos ao acaso, com 13 tratamentos e 4 repetições, para cada cultura. As parcelas foram dimensionadas com 4 linhas de cada cultura, caracterizadas por $3 \mathrm{~m}$ de comprimento e $5 \mathrm{~m}$ de largura, totalizando 15 $\mathrm{m}^{2}$, com $10 \mathrm{~m}^{2}$ centrais de área aplicada. A área total do experimento foi de $4680 \mathrm{~m}^{2}$.

Os tratos culturais realizados na área experimental foram os normais exigidos pela cultura no que diz respeito às adubações e ao controle de pragas e doenças. A adubação foi realizada concomitantemente à semeadura, utilizando-se a fórmula comercial 8-28-16 $(\mathrm{NPK})+0,3 \%$ de Zinco, à base de $250 \mathrm{~kg} \mathrm{ha}^{-1}$. As plantas daninhas que ocorreram, foram capinadas e retiradas manualmente de todos os tratamentos durante todo o período do ensaio.

Os herbicidas nicosulfuron isolado $(60 \mathrm{~g}$ $\mathrm{ha}^{-1}$; formulação comercial Accent $\left.{ }^{\circledR} 80 \mathrm{~g} \mathrm{ha}^{-1}\right)$ e nicosulfuron + atrazine $\left(30 \mathrm{~g}+1500 \mathrm{~g} \mathrm{ha}^{-1}\right.$; formulação comercial Accent ${ }^{\circledR}+$ Atrazinax $500^{\circledR} 40 \mathrm{~g}+3 \mathrm{~L} \mathrm{ha}^{-1}$ ), acrescidos de óleo mineral Assist com 0,3\% v/v, foram aplicados ao solo aos 75, 60, 45, 30, 15 e 7 dias antes da semeadura das culturas, com o intuito de se analisar o efeito residual ("carryover") sobre as plantas cultivadas e de uma testemunha sem herbicida.

As aplicações dos herbicidas foram realizadas com um pulverizador costal com pressão constante $\left(\mathrm{CO}_{2}\right)$ de $45 \mathrm{lb} \mathrm{pol}^{-2}$, provido de tanque com capacidade de dois litros (garrafas descartáveis), e com barra equipada com quatro pontas tipo leque, Teejet XR 110.02, espaçados de 0,5 metros, o que proporcionou volume de calda de $200 \mathrm{~L} \mathrm{ha}^{-1}$. Em todas as aplicações o solo encontrava-se com umidade; a temperatura do ambiente foi sempre menor que $31^{\circ} \mathrm{C}$, a UR ar superior a $55 \%$ e a velocidade do vento inferior a $2 \mathrm{~km} \mathrm{~h}^{-}$ 1 .

A seletividade dos tratamentos às plantas das culturas foi avaliada aos 15, 30 e 45 dias após a semeadura (DDS). Utilizou-se uma escala visual, considerando-se a biomassa e a coloração das plantas tratadas comparadas com as plantas da testemunha, atribuindo-se notas de $0 \%$ a $100 \%$, onde $0 \%$ representou nenhum sintoma de fitotoxicidade e $100 \%$ a morte total das plantas, conforme metodologia proposta por SBCPD (1995). No florescimento das culturas foi avaliada a altura de plantas, sendo os resultados submetidos a analise de variância 
e as médias comparadas pelo teste de Tukey ao nível de $5 \%$ de probabilidade.

\section{Resultados e Discussão}

Os dados médios de fitotoxicidade dos herbicidas e altura de plantas no florescimento das culturas de soja, feijão, algodão, pepino, girassol e arroz, estão apresentados nas Tabelas $1,2,3,4,5$ e 6 . Ressalta-se que em todos os tratamentos ocorreram sintomas de fitotoxicidade inicial (15 DDS), que foram diminuindo gradativamente nas avaliações subsequentes (30 e 45 DDS).
Os sintomas de fitotoxicidade observados já eram esperados pela não seletividade dos herbicidas (atrazine e nicosulfuron) às culturas estudadas. Ressalta-se que a recomendação oficial para o herbicida nicosulfuron, entre a aplicação e o plantio, é de 30 dias para a soja, o feijão, o algodão e o girassol; 45 dias para o arroz e 60 dias para hortaliças como o pepino (Rodrigues \& Almeida, 2005). Entretanto, o residual dos produtos no solo é bastante dependente de condições edafoclimáticas (Ulbrich et al., 2005), o que pode causar diferenças nas pesquisas realizadas em condições variadas.

Tabela 1. Porcentagem de fitotoxicidade em soja aos 15, 30 e 45 dias após a semeadura (DDS), e altura de plantas no florescimento, em relação aos tratamentos nicosulfuron isolado e nicosulfuron + atrazine aplicados 7, 15, 30, 45, 60 e 75 dias antes da semeadura (DAS). FEPSelvíria/MS, 2009.

\begin{tabular}{|c|c|c|c|c|}
\hline \multirow{2}{*}{ TRATAMENTOS } & \multicolumn{3}{|c|}{$\%$ de Fitotoxicidade } & \multirow{2}{*}{$\begin{array}{c}\text { Altura no } \\
\text { florescimento }(\mathrm{cm})\end{array}$} \\
\hline & $15 \mathrm{DDS}$ & $30 \mathrm{DDS}$ & 45 DDS & \\
\hline 01. nicosulfuron + atrazine $\left(30 \mathrm{~g}+1500 \mathrm{~g} \mathrm{ha}^{-1}\right)-75 \mathrm{DAS}$ & 0,2 & 0,0 & 0,0 & $66,0 \mathrm{ab}$ \\
\hline 02. Accent $\left(60 \mathrm{~g} \mathrm{ha}^{-1}\right)-75 \mathrm{DAS}$ & 0,0 & 0,0 & 0,0 & 67,0 a \\
\hline 03. nicosulfuron + atrazine $\left(30 \mathrm{~g}+1500 \mathrm{~g} \mathrm{ha}^{-1}\right)-60 \mathrm{DAS}$ & 1,2 & 0,6 & 0,2 & $66,0 \mathrm{ab}$ \\
\hline 04. Accent $\left(60 \mathrm{~g} \mathrm{ha}^{-1}\right)-60$ DAS & 1,0 & 0,8 & 0,2 & $65,5 \mathrm{ab}$ \\
\hline 05. nicosulfuron + atrazine $\left(30 \mathrm{~g}+1500 \mathrm{~g} \mathrm{ha}^{-1}\right)-45 \mathrm{DAS}$ & 1,8 & 1,0 & 0,2 & 67,0 a \\
\hline 06. Accent $\left(60 \mathrm{~g} \mathrm{ha}^{-1}\right)-45$ DAS & 1,0 & 0,6 & 0,2 & $65,5 \mathrm{ab}$ \\
\hline 07. nicosulfuron + atrazine $\left(30 \mathrm{~g}+1500 \mathrm{~g} \mathrm{ha}^{-1}\right)-30 \mathrm{DAS}$ & 3,6 & 1,6 & 1,6 & $65,5 \mathrm{ab}$ \\
\hline 08. Accent $\left(60 \mathrm{~g} \mathrm{ha}^{-1}\right)-30$ DAS & 2,0 & 1,6 & 1,0 & $66,8 \mathrm{ab}$ \\
\hline 09. nicosulfuron + atrazine $\left(30 \mathrm{~g}+1500 \mathrm{~g} \mathrm{ha}^{-1}\right)-15 \mathrm{DAS}$ & 5,0 & 3,0 & 2,0 & 60,0 \\
\hline 10. Accent $\left(60 \mathrm{~g} \mathrm{ha}^{-1}\right)-15$ dias antes & 3,0 & 2,0 & 1,6 & 66,0 a \\
\hline 11. nicosulfuron + atrazine $\left(30 \mathrm{~g}+1500 \mathrm{~g} \mathrm{ha}^{-1}\right)-07$ DAS & 7,0 & 4,0 & 3,0 & $60,0 \quad b$ \\
\hline 12. Accent $\left(60 \mathrm{~g} \mathrm{ha}^{-1}\right)-07$ DAS & 8,0 & 4,0 & 2,5 & 59,0 \\
\hline 13. Testemunha sem herbicida & 0,0 & 0,0 & 0,0 & $66,0 \mathrm{ab}$ \\
\hline \multicolumn{4}{|c|}{ Média Geral } & 64,64 \\
\hline & \multicolumn{3}{|c|}{ Coeficiente de Variação } & 5,84 \\
\hline
\end{tabular}

Obs: Médias seguidas de letras iguais nas colunas não diferem entre si pelo teste de Tukey (5\%).

Analisando os resultados de fitotoxicidade (15, 30 e 45 DDS) e de altura de plantas no florescimento, assim como os períodos de semeadura $(7,15,30,45,60$ e 75 DAS) após a aplicação dos herbicidas, em que houve danos para as culturas, observou-se que para a cultura da soja houve danos nas aplicações aos 7 e 15 dias antes da semeadura (DAS) para o tratamento nicosulfuron + atrazine e aos 7 dias antes da semeadura (DAS) para o tratamento nicosulfuron isolado. Nas demais épocas de aplicação, os sintomas de fitotoxicidade foram baixos e não houve reduções significativas no crescimento das 
plantas, portanto, o limite de segurança considerado adequado para cultivar de soja Carrera foi de 30 dias para o tratamento com nicosulfuron + atrazine e de 15 dias para o nicosulfuron isolado.

Para a cultura do feijão observou-se que houve danos visuais nas aplicações aos 7 e 15 DAS para os tratamentos nicosulfuron + atrazine e para nicosulfuron isolado (Tabela 2).

Nas demais épocas de aplicação, os sintomas de fitotoxicidade foram baixos e não houve reduções significativas no crescimento das plantas. Portanto, o limite de segurança para a cultura do feijoeiro, variedade carioca, foi de 30 dias para os tratamentos nicosulfuron + atrazine e nicosulfuron isolado.

Para a cultura do algodão observou-se que houve danos nas aplicações aos 7, 15, $30 \mathrm{e}$ 45 DAS para o tratamento nicosulfuron + atrazine e aos 7,15 e 30 DAS para o tratamento nicosulfuron isolado (Tabela 3). Após estes períodos não foram verificados sintomas visuais de fitotoxicidade para as plantas de algodão. Portanto, o limite de segurança para semeadura da cultura do algodoeiro, variedade Delta-Opal, foi de 60 dias para o tratamento nicosulfuron + atrazine e de 45 dias para nicosulfuron isolado.

Tabela 2. Porcentagem de fitotoxicidade em feijão aos 15, 30 e 45 dias após a semeadura (DDS), e altura de plantas no florescimento, em relação aos tratamentos nicosulfuron isolado e nicosulfuron + atrazine aplicados 7, 15, 30, 45, 60 e 75 dias antes da semeadura (DAS). FEPSelvíria/MS, 2009.

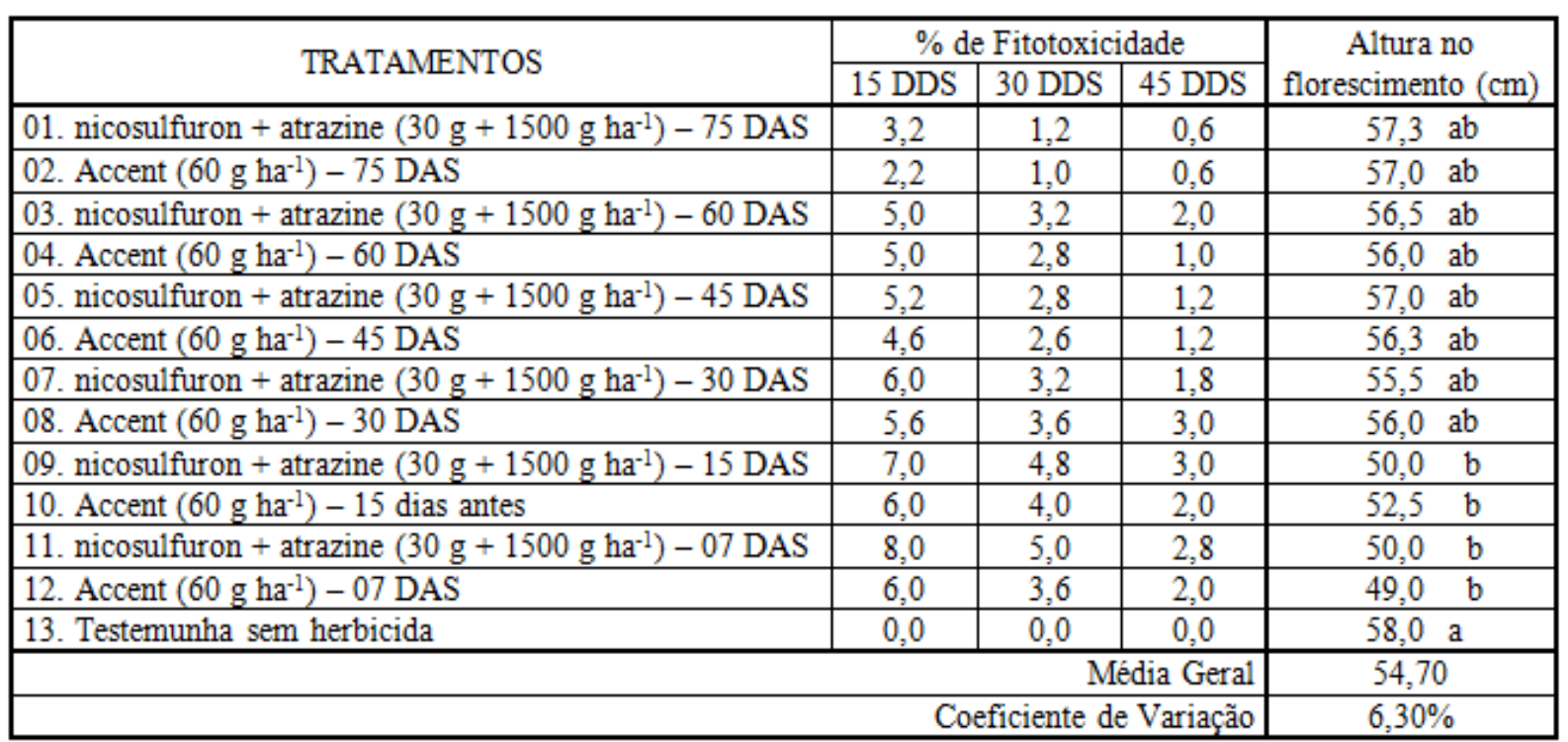

Obs: Médias seguidas de letras iguais nas colunas não diferem entre si pelo teste de Tukey (5\%).

Para o pepino observou-se que houve sintomas visuais de fitotoxicidade nas aplicações aos 7 e 15 DAS para os tratamentos nicosulfuron + atrazine e para nicosulfuron isolado (Tabela 4). Nas demais épocas de aplicação, os sintomas de fitotoxicidade foram baixos e não houve reduções significativas no crescimento das plantas. Portanto, o limite de segurança para cultura do pepino, cultivar Caipira-Esmeralda, foi de 30 dias para os tratamentos nicosulfuron + atrazine $\mathrm{e}$ nicosulfuron isolado. 
Tabela 3. Porcentagem de fitotoxicidade em algodão aos 15, 30 e 45 dias após a semeadura (DDS), e altura de plantas no florescimento, em relação aos tratamentos nicosulfuron isolado e nicosulfuron + atrazine aplicados 7, 15, 30, 45, 60 e 75 dias antes da semeadura (DAS). FEPSelvíria/MS, 2009.

\begin{tabular}{|c|c|c|c|c|}
\hline \multirow{2}{*}{ TRATAMENTOS } & \multicolumn{3}{|c|}{$\%$ de Fitotoxicidade } & \multirow{2}{*}{$\begin{array}{c}\text { Altura no } \\
\text { florescimento }(\mathrm{cm})\end{array}$} \\
\hline & $15 \mathrm{DDS}$ & $30 \mathrm{DDS}$ & $45 \mathrm{DDS}$ & \\
\hline 01. nicosulfuron + atrazine $\left(30 \mathrm{~g}+1500 \mathrm{~g} \mathrm{ha}^{-1}\right)-75$ DAS & 1,2 & 0,6 & 0,2 & 97,5 a \\
\hline 02. Accent $\left(60 \mathrm{~g} \mathrm{ha}^{-1}\right)-75 \mathrm{DAS}$ & 1,6 & 0,8 & 0,2 & 97,3 a \\
\hline 03. nicosulfuron + atrazine $\left(30 \mathrm{~g}+1500 \mathrm{~g} \mathrm{ha}^{-1}\right)-60 \mathrm{DAS}$ & 3,2 & 2,0 & 1,0 & $95,0 \mathrm{ab}$ \\
\hline 04. Accent $\left(60 \mathrm{~g} \mathrm{ha}^{-1}\right)-60$ DAS & 2,6 & 1,8 & 0,8 & 97,8 a \\
\hline 05. nicosulfuron + atrazine $\left(30 \mathrm{~g}+1500 \mathrm{~g} \mathrm{ha}^{-1}\right)-45$ DAS & 5,6 & 2,6 & 1,2 & 90,8 bc \\
\hline 06. Accent $\left(60 \mathrm{~g} \mathrm{ha}^{-1}\right)-45$ DAS & 4,6 & 2,6 & 1,0 & 98,5 a \\
\hline 07. nicosulfuron + atrazine $\left(30 \mathrm{~g}+1500 \mathrm{~g} \mathrm{ha}^{-1}\right)-30 \mathrm{DAS}$ & 11,0 & 6,6 & 4,0 & $87,5 \mathrm{c}$ \\
\hline 08. Accent $\left(60 \mathrm{~g} \mathrm{ha}^{-1}\right)-30$ DAS & 6,0 & 4,0 & 1,8 & $92,0 \mathrm{~b}$ \\
\hline 09. nicosulfuron + atrazine $\left(30 \mathrm{~g}+1500 \mathrm{~g} \mathrm{ha}^{-1}\right)-15 \mathrm{DAS}$ & 13,0 & 10,0 & 6,0 & $85,3 \mathrm{c}$ \\
\hline 10. Accent $\left(60 \mathrm{~g} \mathrm{ha}^{-1}\right)-15$ dias antes & 8,0 & 5,0 & 3,0 & $92,3 \mathrm{~b}$ \\
\hline 11. nicosulfuron + atrazine $\left(30 \mathrm{~g}+1500 \mathrm{~g} \mathrm{ha}^{-1}\right)-07 \mathrm{DAS}$ & 16,0 & 11,0 & 7,0 & $85,0 \mathrm{c}$ \\
\hline 12. Accent $\left(60 \mathrm{~g} \mathrm{ha}^{-1}\right)-07$ DAS & 10,0 & 8,0 & 6,0 & $87,0 \mathrm{c}$ \\
\hline 13. Testemunha sem herbicida & 0,0 & 0,0 & 0,0 & $100,0 \quad \mathrm{a}$ \\
\hline \multicolumn{4}{|c|}{ Média Geral } & 91,69 \\
\hline & \multicolumn{3}{|c|}{ Coeficiente de Variação } & $3,75 \%$ \\
\hline
\end{tabular}

Obs: Médias seguidas de letras iguais nas colunas não diferem entre si pelo teste de Tukey (5\%).

Para o girassol observou-se que houve danos nas aplicações aos 7 e 15 DAS para os tratamentos nicosulfuron + atrazine e para nicosulfuron isolado. Nas demais épocas de aplicação, os sintomas de fitotoxicidade foram baixos e não houve reduções significativas no crescimento das plantas. Portanto, o limite de segurança para o girassol, híbrido Dow M734G3, foi de 30 dias para os tratamentos nicosulfuron + atrazine e nicosulfuron isolado.

Tabela 4. Porcentagem de fitotoxicidade em pepino aos 15, 30 e 45 dias após a semeadura (DDS), e altura de plantas no florescimento, em relação aos tratamentos nicosulfuron isolado e nicosulfuron + atrazine aplicados 7, 15, 30, 45, 60 e 75 dias antes da semeadura (DAS). FEPSelvíria/MS, 2009.

\begin{tabular}{|c|c|c|c|c|}
\hline \multirow{2}{*}{ TRATAMENTOS } & \multicolumn{3}{|c|}{$\%$ de Fitotoxicidade } & \multirow{2}{*}{$\begin{array}{c}\text { Altura no } \\
\text { florescimento }(\mathrm{cm})\end{array}$} \\
\hline & $15 \mathrm{DDS}$ & $30 \mathrm{DDS}$ & $45 \mathrm{DDS}$ & \\
\hline 01. nicosulfuron + atrazine $\left(30 \mathrm{~g}+1500 \mathrm{~g} \mathrm{ha}^{-1}\right)-75 \mathrm{DAS}$ & 2,0 & 1,0 & 0,2 & $51,0 \quad \mathrm{a}$ \\
\hline 02. Accent $\left(60 \mathrm{~g} \mathrm{ha}^{-1}\right)-75$ DAS & 1,8 & 0,8 & 0,2 & 52,0 a \\
\hline 03. nicosulfuron + atrazine $\left(30 \mathrm{~g}+1500 \mathrm{~g} \mathrm{ha}^{-1}\right)-60 \mathrm{DAS}$ & 2,2 & 1,8 & 0,8 & $50,5 \mathrm{ab}$ \\
\hline 04. Accent $\left(60 \mathrm{~g} \mathrm{ha}^{-1}\right)-60$ DAS & 2,0 & 1,0 & 0,6 & $50,5 \mathrm{ab}$ \\
\hline 05 . nicosulfuron + atrazine $\left(30 \mathrm{~g}+1500 \mathrm{~g} \mathrm{ha}^{-1}\right)-45 \mathrm{DAS}$ & 2,6 & 1,2 & 1,0 & $50,0 \mathrm{ab}$ \\
\hline 06. Accent $\left(60 \mathrm{~g} \mathrm{ha}^{-1}\right)-45$ DAS & 2,0 & 1,0 & 0,8 & $50,5 \mathrm{ab}$ \\
\hline 07. nicosulfuron + atrazine $\left(30 \mathrm{~g}+1500 \mathrm{~g} \mathrm{ha}^{-1}\right)-30 \mathrm{DAS}$ & 4,0 & 2,0 & 1,2 & $49,3 \mathrm{ab}$ \\
\hline 08. Accent $\left(60 \mathrm{~g} \mathrm{ha}^{-1}\right)-30$ DAS & 3,0 & 1,2 & 0,8 & $49,5 \mathrm{ab}$ \\
\hline 09. nicosulfuron + atrazine $\left(30 \mathrm{~g}+1500 \mathrm{~g} \mathrm{ha}^{-1}\right)-15 \mathrm{DAS}$ & 5,0 & 3,0 & 1,8 & 46,3 bc \\
\hline 10. Accent $\left(60 \mathrm{~g} \mathrm{ha}^{-1}\right)-15$ dias antes & 4,0 & 2,6 & 1,6 & 45,5 bc \\
\hline 11. nicosulfuron + atrazine $\left(30 \mathrm{~g}+1500 \mathrm{~g} \mathrm{ha}^{-1}\right)-07$ DAS & 6,2 & 5,0 & 3,0 & 45,0 bc \\
\hline 12. Accent $\left(60 \mathrm{~g} \mathrm{ha}^{-1}\right)-07 \mathrm{DAS}$ & 4,8 & 3,8 & 2,2 & $44,0 \mathrm{~b}$ \\
\hline 13. Testemunha sem herbicida & 0,0 & 0,0 & 0,0 & 52,0 a \\
\hline \multicolumn{4}{|c|}{ Média Geral } & 48,93 \\
\hline & \multicolumn{3}{|c|}{ Coeficiente de Variação } & $6,48 \%$ \\
\hline
\end{tabular}

Obs: Médias seguidas de letras iguais nas colunas não diferem entre si pelo teste de Tukey (5\%). 
Tabela 5. Porcentagem de fitotoxicidade em girassol aos 15, 30 e 45 dias após a semeadura (DDS), e altura de plantas no florescimento, em relação aos tratamentos nicosulfuron isolado e nicosulfuron + atrazine aplicados 7, 15, 30, 45, 60 e 75 dias antes da semeadura (DAS). FEPSelvíria/MS, 2009.

\begin{tabular}{|c|c|c|c|c|}
\hline \multirow{2}{*}{ TRATAMENTOS } & \multicolumn{3}{|c|}{$\%$ de Fitotoxicidade } & \multirow{2}{*}{$\begin{array}{c}\text { Altura no } \\
\text { florescimento }(\mathrm{cm})\end{array}$} \\
\hline & $15 \mathrm{DDS}$ & 30 DDS & $45 \mathrm{DDS}$ & \\
\hline 01. nicosulfuron + atrazine $\left(30 \mathrm{~g}+1500 \mathrm{~g} \mathrm{ha}^{-1}\right)-75 \mathrm{DAS}$ & 3,0 & 1,8 & 0,8 & 115,5 a \\
\hline 02. Accent $\left(60 \mathrm{~g} \mathrm{ha}^{-1}\right)-75 \mathrm{DAS}$ & 2,8 & 1,2 & 0,2 & 115,0 a \\
\hline 03. nicosulfuron + atrazine $\left(30 \mathrm{~g}+1500 \mathrm{~g} \mathrm{ha}^{-1}\right)-60 \mathrm{DAS}$ & 3,2 & 2,0 & 1,0 & $114,3 \mathrm{ab}$ \\
\hline 04. Accent $\left(60 \mathrm{~g} \mathrm{ha}^{-1}\right)-60 \mathrm{DAS}$ & 2,8 & 1,2 & 0,2 & 115,0 a \\
\hline 05. nicosulfuron + atrazine $\left(30 \mathrm{~g}+1500 \mathrm{~g} \mathrm{ha}^{-1}\right)-45$ DAS & 3,6 & 2,0 & 1,0 & 115,3 a \\
\hline 06. Accent $\left(60 \mathrm{~g} \mathrm{ha}^{-1}\right)-45$ DAS & 3,0 & 1,2 & 0,6 & 116,3 a \\
\hline 07. nicosulfuron + atrazine $\left(30 \mathrm{~g}+1500 \mathrm{~g} \mathrm{ha}^{-1}\right)-30 \mathrm{DAS}$ & 4,0 & 2,8 & 1,0 & 116,8 a \\
\hline 08. Accent $\left(60 \mathrm{~g} \mathrm{ha}^{-1}\right)-30$ DAS & 3,2 & 2,0 & 0,6 & 116,5 a \\
\hline 09. nicosulfuron + atrazine $\left(30 \mathrm{~g}+1500 \mathrm{~g} \mathrm{ha}^{-1}\right)-15 \mathrm{DAS}$ & 6,0 & 2,8 & 1,2 & $112,5 \mathrm{bc}$ \\
\hline 10. Accent $\left(60 \mathrm{~g} \mathrm{ha}^{-1}\right)-15$ dias antes & 6,6 & 3,6 & 2,0 & $112,0 \mathrm{bc}$ \\
\hline 11. nicosulfuron + atrazine $\left(30 \mathrm{~g}+1500 \mathrm{~g} \mathrm{ha}^{-1}\right)-07$ DAS & 9,0 & 6,0 & 5,0 & 112,0 bc \\
\hline 12. Accent $\left(60 \mathrm{~g} \mathrm{ha}^{-1}\right)-07$ DAS & 7,0 & 3,0 & 2,0 & $108,3 \mathrm{c}$ \\
\hline 13. Testemunha sem herbicida & 0,0 & 0,0 & 0,0 & 116,0 a \\
\hline \multicolumn{4}{|c|}{ Média Geral } & 114,27 \\
\hline \multicolumn{4}{|c|}{ Coeficiente de Variação } & $4,11 \%$ \\
\hline
\end{tabular}

Obs: Médias seguidas de letras iguais nas colunas não diferem entre si pelo teste de Tukey ( $5 \%)$.

Tabela 6. Porcentagem de fitotoxicidade em arroz aos 15, 30 e 45 dias após a semeadura (DDS), e altura de plantas no florescimento, em relação aos tratamentos nicosulfuron isolado e nicosulfuron + atrazine aplicados 7, 15, 30, 45, 60 e 75 dias antes da semeadura (DAS). FEPSelvíria/MS, 2009.

\begin{tabular}{|c|c|c|c|c|}
\hline \multirow{2}{*}{ TRATAMENTOS } & \multicolumn{3}{|c|}{$\%$ de Fitotoxicidade } & \multirow{2}{*}{$\begin{array}{c}\text { Altura no } \\
\text { florescimento }(\mathrm{cm})\end{array}$} \\
\hline & $15 \mathrm{DDS}$ & $30 \mathrm{DDS}$ & $45 \mathrm{DDS}$ & \\
\hline 01. nicosulfuron + atrazine $\left(30 \mathrm{~g}+1500 \mathrm{~g} \mathrm{ha}^{-1}\right)-75 \mathrm{DAS}$ & 3,0 & 2,2 & 1,6 & 62,5 a \\
\hline 02. Accent $\left(60 \mathrm{~g} \mathrm{ha}^{-1}\right)-75$ DAS & 2,8 & 2,0 & 1,2 & $61,5 \mathrm{ab}$ \\
\hline 03. nicosulfuron + atrazine $\left(30 \mathrm{~g}+1500 \mathrm{~g} \mathrm{ha}^{-1}\right)-60 \mathrm{DAS}$ & 4,5 & 3,2 & 1,8 & $61,0 \quad a b$ \\
\hline 04. Accent $\left(60 \mathrm{~g} \mathrm{ha}^{-1}\right)-60$ DAS & 3,0 & 2,2 & 1,2 & $62,0 \mathrm{ab}$ \\
\hline 05. nicosulfuron + atrazine $\left(30 \mathrm{~g}+1500 \mathrm{~g} \mathrm{ha}^{-1}\right)-45$ DAS & 5,0 & 3,6 & 2,2 & $56,5 \mathrm{~b}$ \\
\hline 06. Accent $\left(60 \mathrm{~g} \mathrm{ha}^{-1}\right)-45$ DAS & 5,0 & 3,0 & 2,0 & 55,0 \\
\hline 07. nicosulfuron + atrazine $\left(30 \mathrm{~g}+1500 \mathrm{~g} \mathrm{ha}^{-1}\right)-30$ DAS & 6,0 & 5,0 & 4,0 & 54,0 \\
\hline 08. Accent $\left(60 \mathrm{~g} \mathrm{ha}^{-1}\right)-30$ DAS & 7,6 & 5,2 & 4,8 & 54,0 \\
\hline 09. nicosulfuron + atrazine $\left(30 \mathrm{~g}+1500 \mathrm{~g} \mathrm{ha}^{-1}\right)-15 \mathrm{DAS}$ & 7,2 & 5,6 & 4,6 & 53,0 \\
\hline 10. Accent $\left(60 \mathrm{~g} \mathrm{ha}^{-1}\right)-15$ dias antes & 10,0 & 7,2 & 6,0 & 52,0 \\
\hline 11. nicosulfuron + atrazine $\left(30 \mathrm{~g}+1500 \mathrm{~g} \mathrm{ha}^{-1}\right)-07$ DAS & 9,0 & 6,2 & 3,0 & 50,0 \\
\hline 12. Accent $\left(60 \mathrm{~g} \mathrm{ha}^{-1}\right)-07$ DAS & 14,0 & 9,0 & 8,0 & $45,8 \quad \mathrm{c}$ \\
\hline 13. Testemunha sem herbicida & 0,0 & 0,0 & 0,0 & 63,0 a \\
\hline \multicolumn{4}{|c|}{ Média Geral } & 56,88 \\
\hline & \multicolumn{3}{|c|}{ Coeficiente de Variação } & $7,42 \%$ \\
\hline
\end{tabular}

Obs: Médias seguidas de letras iguais nas colunas não diferem entre si pelo teste de Tukey (5\%). 
Para o arroz observou-se que houve danos nas aplicações aos 7, 15, 30 e 45 DAS para os tratamentos nicosulfuron + atrazine $\mathrm{e}$ para nicosulfuron isolado. Nas demais épocas de aplicação, os sintomas de fitotoxicidade foram baixos e não houve reduções significativas no crescimento das plantas. Portanto, o limite de segurança para a variedade de arroz Primavera foi de 60 dias para os tratamentos nicosulfuron + atrazina $e$ para nicosulfuron isolado.

\section{Conclusões}

Considerando-se os resultados obtidos concluiu-se que as culturas do feijão (Carioca), pepino (Caipira-Esmeralda) e girassol (Dow M734-G3) podem ser semeadas após 30 dias da aplicação de nicosulfuron + atrazine e nicosulfuron isolado.

A cultivar de soja Carrera pode ser semeada 15 e 30 dias após a aplicação de nicosulfuron + atrazine e nicosulfuron isolado, respectivamente. $\mathrm{O}$ algodão (Delta-Opal) após 60 e 45 dias da aplicação de nicosulfuron + atrazine e nicosulfuron isolado, respectivamente.

Já o arroz (Primavera) pode ser semeado após 60 dias da aplicação de nicosulfuron + atrazine e nicosulfuron isolado.

\section{Referências}

ANDREI, E. Compêndio dos defensivos agrícolas. $7^{\text {a }}$ Ed. Rev. e Atualizada, São Paulo - SP: Organização Andrei Editora Ltda., 2005. $1141 \mathrm{p}$.

CARVALHO, F.T.; GALLI, A.J.B. Nova formulação de alachlor na seletividade e no controle de plantas daninhas na cultura do milho. In: CONGRESSO BRASILEIRO DE HERBICIDAS E PLANTAS DANINHAS, 19,
1993 Londrina, PR. Resumos... Londrina: SBHED, 1993. p.131.

FANCELLI, A.L.; DOURADO NETO, D. Produção de milho. Guaíba: Agropecuária, 2000. 360 p.

IBGE - Instituto Brasileiro de Geografia e Estatística. Levantamento Sistemático da Produção Agrícola - Abril/Mai - 2009. Disponível em <http://www.ibge.gov.br/home /estatistica/indicadores/agropecuária/lspa/lspa 200905_3.shtm>. Acesso em: 18/10/2010

LOPEZ-OVEJERO, R.F. et al. Seletividade de herbicidas para a cultura de milho (Zea mays) aplicados em diferentes estádios fenológicos da cultura. Planta Daninha, v.21, n.3, p.413419, 2003.

LORENZI, H. et al. Manual de Identificação e controle de plantas daninhas: plantio direto e convencional, $6^{\mathrm{a}}$ Ed., Nova Odessa, SP: Plantarum, 2006. 339 p.

RODRIGUES, B.N.; ALMEIDA, F.S. Guia de herbicidas. $5^{\text {a }}$ Ed., Londrina: Edição dos Autores, 2005. 592 p.

SOCIEDADE BRASILEIRA DA CIÊNCIA DAS PLANTAS DANINHAS (SBCPD). Procedimentos para instalação, avaliação e análise de experimentos com herbicidas. Londrina: SBCPD, 1995. 42p.

SILVA, M.S.J. et al. Estudo do herbicida nicosulfuron no controle de plantas daninhas na cultura do milho (Zea mays L.). In: CONGRESSO BRASILEIRO DE HERBICIDAS E PLANTAS DANINHAS, 19, 1993, Londrina, PR. Resumos... Londrina: SBHED, 1993. p.148-149.

ULBRICH, A.V.; SOUZA, J.R.P.; SHANER, D. Persistence and carryover effect of imazapic and imazapyr in Brazilian cropping systems. Weed Technology, v.9, n.4, p.986$991,2005$. 
VELINI, E. D. et al. Eficiência de herbicidas pré e pós emergentes iniciais recomendados para a cultura do milho, no controle de plantas daninhas e avaliação de efeitos dos mesmos sobre a produtividade da cultura. In: CONGRESSO BRASILEIRO DE HERBICIDAS E PLANTAS DANINHAS, 19, 1993, Londrina, PR. Resumos... Londrina: SBHED, 1993. p. 141-142. 